\title{
On interplay between excitability and geometry
}

\author{
Andrew Adamatzky \\ Unconventional Computing Laboratory, \\ University of the West of England, Bristol BS16 1QY, UK
}

\begin{abstract}
A commonly accepted feature of an excitable medium is that a local excitation leads to a propagation of circular or spiral excitation wave-fronts. This is indeed the case in fully excitable medium. However, with a decrease of an excitability localised wave-fragments emerge and propagate ballistically. Using FitzhHugh-Nagumo model we numerically study how excitation wave-fronts behave in a geometrically constrained medium and how the wave-fronts explore a random planar graph. We uncover how excitability controls propagation of excitation in angled branches, influences arrest of excitation entering a sudden expansion, and determines patterns of traversing of a random planar graph by an excitation waves.
\end{abstract}

Keywords: excitable medium, wave-fronts, FitzHugh-Nagumo model

\section{Introduction}

Excitation is essential property of all living creatures, from bacteria [31, protists [15, 24, 11], fungi [32] and plants [39, 19, 45] to vertebrates [25, 7, 34, 14]. Waves of excitation could be also found in various physical [26, 40, 38, 22], chemical [9, 43, 44] and social systems [17, 16]. A study of a propagation of excitation wave-fronts in geometrically constrained media also brings a high value in future and emergent computing technologies because there is a substantial number of theoretical and experimental laboratory prototypes of unconventional computing devices based on the interaction of the wavefronts [2, 3].

Interactions of wave-fronts with inhomogeneities of a medium and geometrical constrains of the medium has been studied for the case of a fully excitable medium, especially in the context of myocardiuam and formation of spiral waves due to inhomogeneities [21, 28, 10, 13, 37, 30, 29, 27, 42, 46, 41, 20. Particularly interesting results include cancellation of excitation wave-fronts in the narrowing areas [27], annihilation or delay of wave-fronts propagating along narrow channel when entering a sudden expansion [28, 13. We reconsider these topics and introduce several new ones in present paper.

A key feature of present study is that we consider behaviour of the medium at a wide range of excitability: from classical fully excitable medium with target waves to sub-excitable medium with localised wave-fragments to non-excitable medium. In numerical experiments we demonstrate dependence of an angle of a branching channel which a wave can enter on the excitability of the medium. We show how the excitability affects arresting of the wave on entering an expansion. We also analyse an extend of a random planar graph exploration by wave-fronts for various values of excitability.

\section{The model}

FitzHugh-Nagumo (FHN) equations [18, 33, 35] give us a qualitative approximation of Hodgkin-Huxley model [8] of electrical activity of living cells:

$$
\begin{aligned}
& \frac{\partial v}{\partial t}=c_{1} u(u-a)(1-u)-c_{2} u v+I+D_{u} \nabla^{2} \\
& \frac{\partial v}{\partial t}=b(u-v)
\end{aligned}
$$


where $u$ is a value of a trans-membrane potential, $v$ is a variable accountable for a total slow ionic current, or a recovery variable responsible for a slow negative feedback, $I$ is a value of external stimulation current. Current through intra-cellular spaces is approximated by $D_{u} \nabla^{2}$, where $D_{u}$ is a conductance. Detailed explanations of the 'mechanics' of the model are provided in [36], here we repeat some insights. The term $D_{u} \nabla^{2} u$ governs a passive spread of the current. The terms $c_{2} u(u-a)(1-u)$ and $b(u-v)$ describe the ionic currents. The term $u(u-a)(1-u)$ has two stable fixed points $u=0$ and $u=1$ and one unstable point $u=a$, where $a$ is a threshold of an excitation. We integrated the system using Euler method with five-node Laplace operator, time step $\Delta t=0.015$ and grid point spacing $\Delta x=2$, other parameters were $D_{u}=1, a=0.13, b=0.013, c_{1}=0.26$, we controlled excitability of the medium by varying $c_{2}$ from 0.09 (fully excitable) to 0.013 (non excitable). Boundaries are considered to be impermeable: $\partial u / \partial \mathbf{n}=0$, where $\mathbf{n}$ is a vector normal to the boundary. The numerical integration code, written in Processing was inspired by [23, 35, 36. Time-lapse snapshots provided in the paper were recorded at every $150^{\text {th }}$ time step, and we display sites with $u>0.04$; videos supplementing figures were produced by saving a frame of the simulation every $100^{\text {th }}$ step of the numerical integration and assembling them in the video with play rate $30 \mathrm{fps}$. Videos are available at [1].

\section{Results}

Propagation of a wave-front in a 'free' space. Let us have a brief look at the wave propagation in a 'free' space for selected values of excitability $c_{2}$ (Fig. 1). In a fully excitable medium, $c_{2}=0.1$ we observe a circular wave propagating from a single source of stimulation (Fig. 1(a)). Since excitability reaches $c_{2}=0.11$ a stimulation evokes not a circular wave but a wave-fragment. The fragment rapidly expands in Fig. 1(b), The time necessary for a wave-fragment to lose its stability and start expanding increases with decrease of excitability (Fig. $1(\mathrm{c})-1(\mathrm{f})$. The excitation shows soliton-like behaviour with a wave-fragment staying compact for hundreds of thousands of iterations for $c_{2}=0.115092$ (Fig. 1(g)) and $c_{2}=0.115092$ (Fig. 1(h)). For $c_{2}>0.115094$ wave-fragments collapse rapidly.

Speed of a wave-front propagation in a channel. As seen in Fig. 1(i), excitation wave-front collapses after nearly $18.5 \cdot 10^{3}$ iterations for $c_{2}=0.115095$, yet when geometrically restricted to a channel of a conducive material, width 20 nodes, the excitation steadily propagates for $c_{2}$ up to 0.1265 (Fig. 1(k) . Geometrical constraining of an excitation enhances lifetime of wave-fragments at lower values of excitability however does not prevent wave-fragments from slowing down (Fig. 1(1)]. A crude linear dependence of a speed $v$ on excitability $c_{2}$ is $v\left(c_{2}\right)=0.027041+(-0.11297) \cdot c_{2}$ and more accurate cubic one is $v\left(c_{2}\right)=0.21425+(-5.4905) \cdot c_{2}+51.057 \cdot c_{2}^{2}+(-160.27) \cdot c_{2}^{3}$.

Wave profile. What happens with a wave profile with decrease of excitability? As illustrated in Fig. $1(\mathrm{~m})$, the increase of $c_{2}$ from 0.1 to 0.12 to 0.126 causes decrease in amplitude. A width of the wave-front does not seem to be affected by the excitability, as seen in Fig. 1(m). However, due to a lower amplitude and a narrower shape the wave-fronts are visualised as narrow for higher values of $c_{2}$.

Excitation propagation into angled branches. Assume a wave-front propagates along a central channel, 20 nodes wide, from which other channels are branching our at various angles. A width of each lateral channel is 20 nodes. How would excitability of the medium affect an ability of the wave-front to enter a later channel branching at a specified angle? To find the answer we designed a template with 15 lateral channels branching out the central channel at angles (Fig. 2(a) from $20^{\circ}$ to $170^{\circ}$ with increment $10^{\circ}$. A distance between two adjacent lateral channels is 60 nodes.

Exemplar time lapses of excitation propagating in the template for various values of $c_{2}$ is shown in Fig. 2(b) 2(i), Excitation enters all lateral channels till $c_{2}=0.1$ (Fig. 2(b) but fails to enter the channel branching out at $170^{\circ}$ when excitability is $c_{2}=0.104$ (Fig. 2(c)). With further increase of $c_{2}$ less channels branching out at obtuse angles remain unexcited (Fig. 2(d)]. At excitability level $c_{2}=0.111$ the excitation fails to enter $90^{\circ}$ channel (Fig. 2(e) . After further decrease of excitability through $c_{2}=118$ (Fig. 2(f) and $c_{2}=0.120$ (Fig. 2(g) $)$ to $c_{2}=0.122$ (Fig. 2(g) excitation fails to propagate along the central channel at $c_{2}=0.126$. 


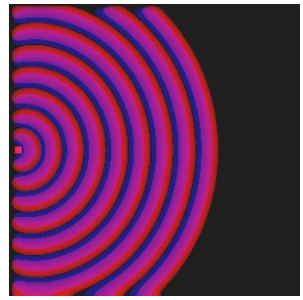

(a) $c_{2}=0.1$

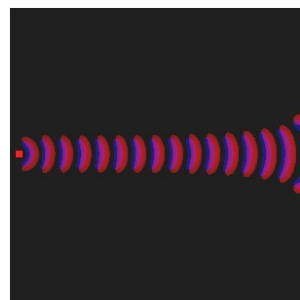

(f) $c_{2}=0.11509$

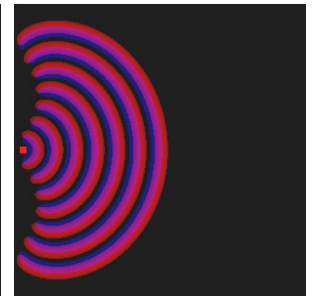

(b) $c_{2}=0.11$

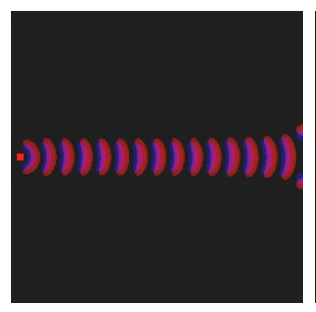

(g) $c_{2}=0.115092$

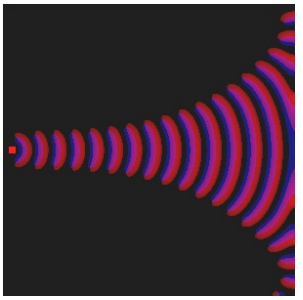

(c) $c_{2}=0.115$

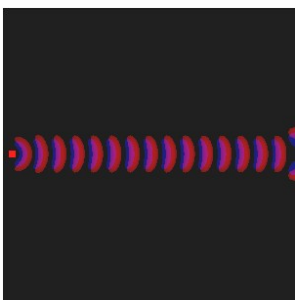

(h) $c_{2}=0.115093$

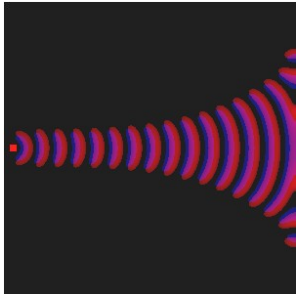

(d) $c_{2}=0.11505$

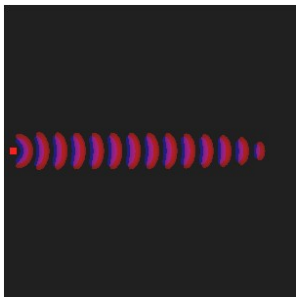

(i) $c_{2}=0.115095$

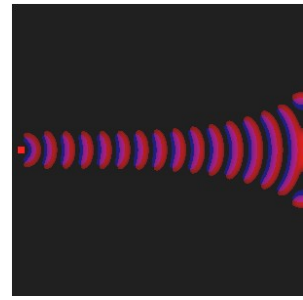

(e) $c_{2}=0.11508$

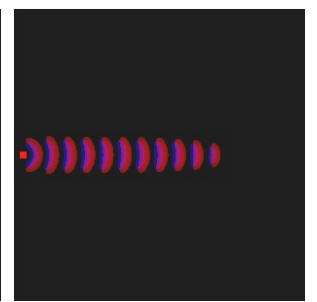

(j) $c_{2}=0.11510$

(k) $c_{2}=0.126$

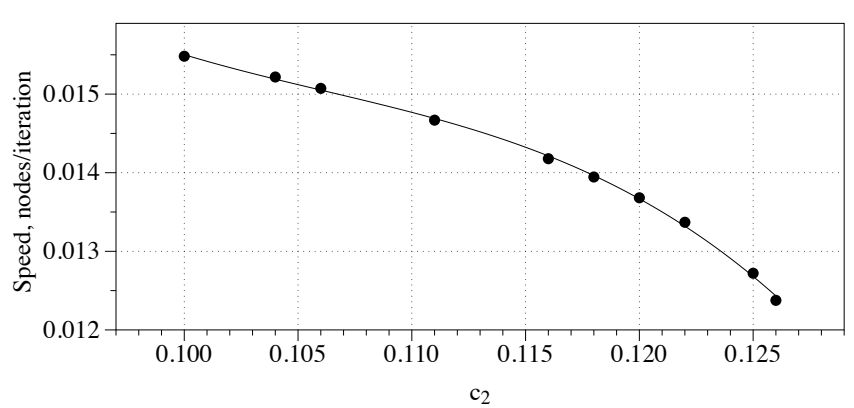

(1)

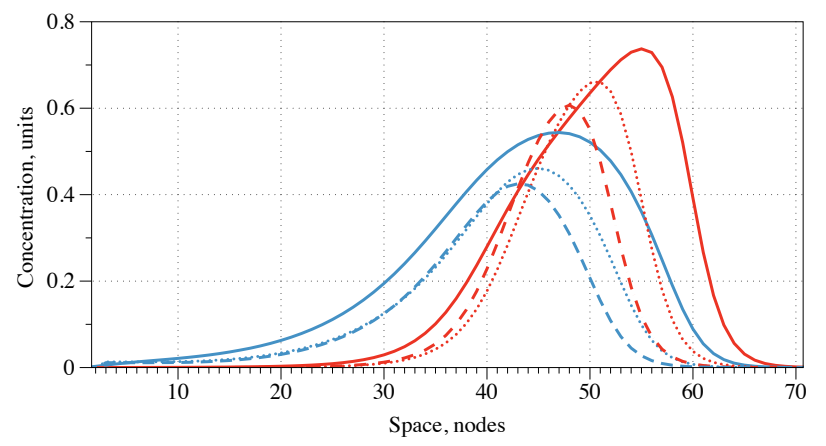

(m)

Figure 1: Propagation of excitation in a free space $(\mathrm{a}-\mathrm{j})$ and a channel of $1700 \times 20$ nodes $(\mathrm{k})$. Each picture shows not several waves but a configuration of a single wave displayed on the same image every $100^{\text {th }}$ iterations. (l) Time lapse of a single wave-front propagating in a channel for $c_{2}=0.126$. (m) Cross cut of the wave-front profile. Concentration of $u$ is shown by red, and $v$ by blue. Solid lines represent the profile of the wave-front for $c_{2}=0.1$, dotted line for $c_{2}=0.12$ and dashed line for $c_{2}=0.126$. Time lapses $(\mathrm{a}-\mathrm{j})$ are at 0.3 scale of the real size, and $(\mathrm{k})$ at 0.27 scale. 


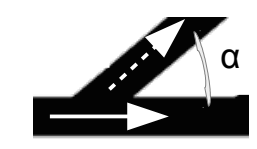

(a)

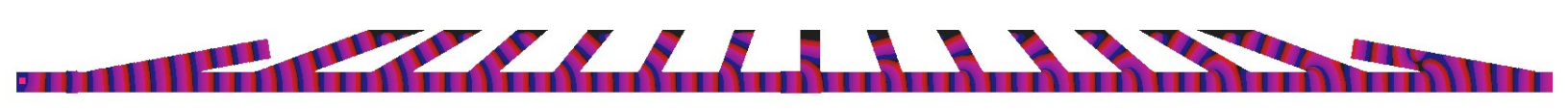

(b) $c_{2}=0.100$

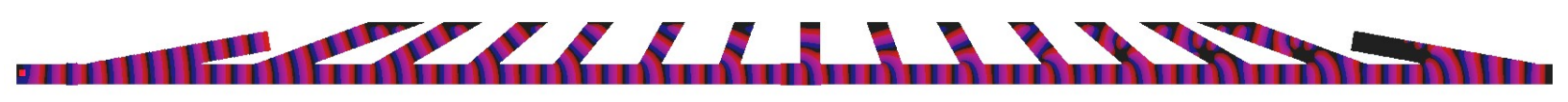

(c) $c_{2}=0.104$

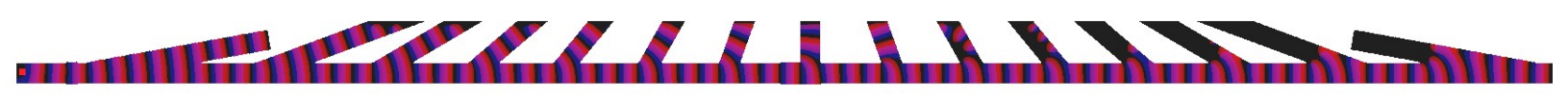

(d) $c_{2}=0.106$

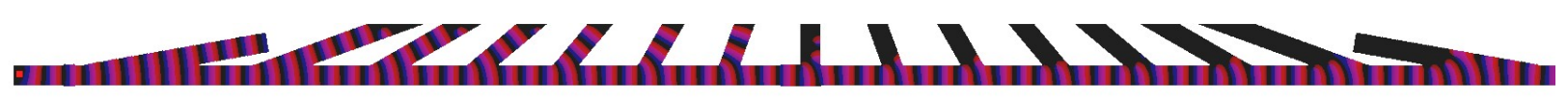

(e) $c_{2}=0.111$

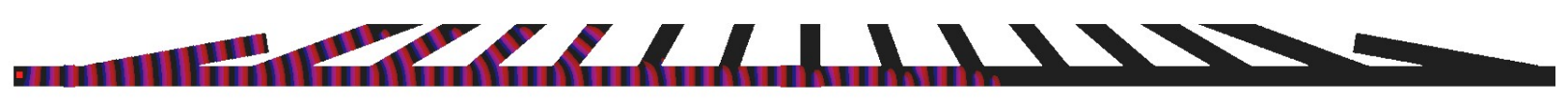

(f) $c_{2}=0.118$

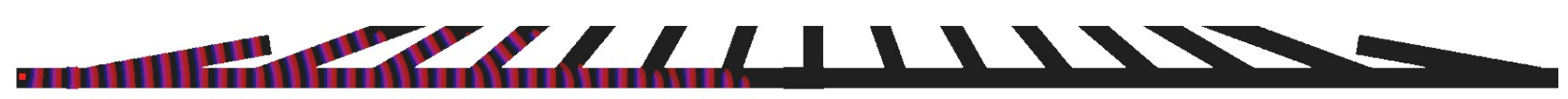

(g) $c_{2}=0.120$

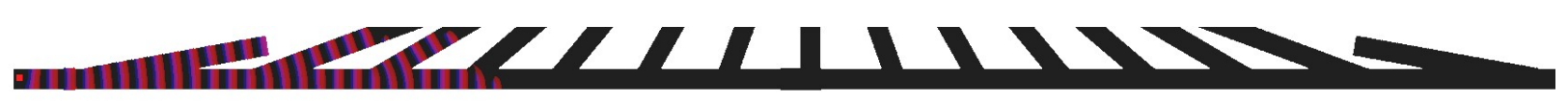

(h) $c_{2}=0.122$

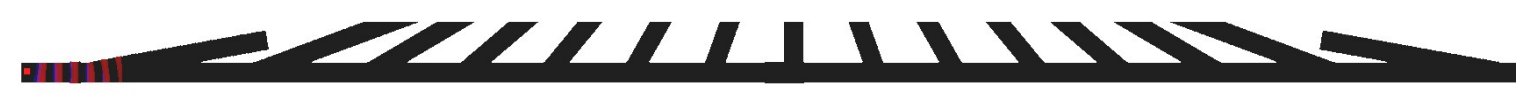

(i) $c_{2}=0.126$

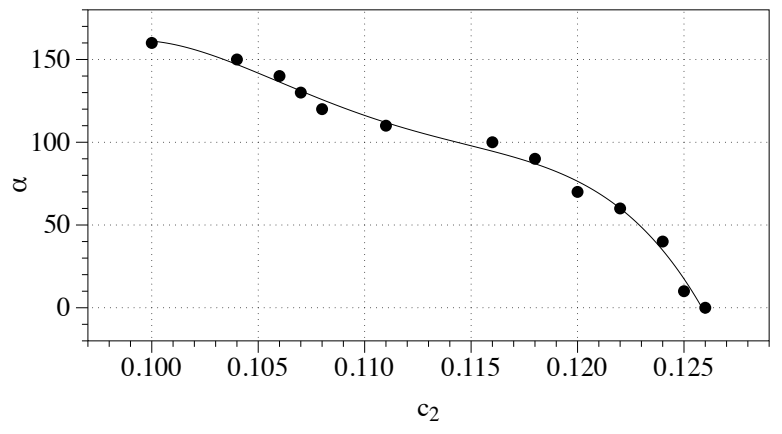

(j)

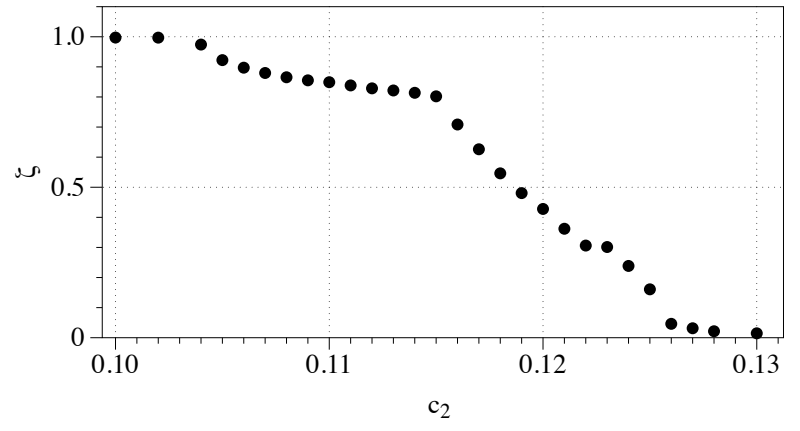

$(\mathrm{k})$

Figure 2: Excitability affects a wave-front propagation into angled branches. (a) A representation of the angle $\alpha$. Solid arrow shows direction of propagation of the wave-front in the central channel, dashed arrow shows direction of propagation of the wave-front entering the angled branch. (b-i) Exemplary time lapses of experiments. All channels have width 20 nodes. (j) Angle $\alpha$ of a lateral channel being entered by excitation wave versus $c_{2}$. Data points are shown by solid discs, polynomial approximations by line. (k) Coverage $\zeta$ versus non-excitability $c_{2}$. Time lapses (b-i) are at 0.27 scale of their real size. See videos in [1]. 
The largest angle $\alpha$ of a lateral channel entered by an excitation decreases polynomially with increase of $c_{2}: \alpha\left(c_{2}\right)=352532+\left(-1.2716 \cdot 10^{7}\right) \cdot c_{2}+1.7136 \cdot 10^{8} \cdot c_{2}^{2}+\left(-1.0227 \cdot 10^{9}\right) \cdot x_{2}^{c}+2.2823 \cdot 10^{9} \cdot c_{2}^{4}$ (Fig. 2(j) . In Fig. 2(k) we give a fine-grained characterisation of the dynamic using the coverage $\zeta$. The coverage $\zeta$ is a ratio of nodes excited at least once during the simulation to a total number of nodes in the template. Three critical values of $c_{2}$ are evident in Fig. 2(k); $c_{2}=0.104$ (wave fails to enter $170^{\circ}$ lateral channel), $c_{2}=0.116$ (wave fails to enter $80^{\circ}$ channel) and $c_{2}=0.126$ (wave fails to propagate in the central channel).

Arrest of an excitation wave-front on its entry into an expansion. To analyse how excitability affects an arrest of a wave-front on entering expansion we prepared 15 templates as follows. Each template is comprised of two rectangles $25 \times 21$ nodes connected by a channel 460 nodes long. Width of the channel was varied from 6 nodes to 20 nodes. We initiated an excitation in the bottom expansion, and allowed the excitation wave to propagate along the narrow channel and into the top expansion. Exemplar time lapses of experiments are shown in Fig. 3(a), 3(b), 3(c), Let $w$ is the smallest width of a channel from which the excitation propagates into the extension reservoir. The dependence of $w$ on $c_{2}$ is shown in Fig. 3(d). The following piece-wise linear approximation is valid: $w\left(c_{2}\right)=2 \cdot 10^{2} \cdot c_{2}-10$, if $c_{2} \in\left[0.08,0.125\left[\right.\right.$ and $w\left(c_{2}\right)=2 \cdot 10^{3} \cdot c_{2}-234$, if $c_{2} \in[0.125,0.127]$.

Exploration of a random plant graph by excitation wave-fronts. A random planar graph $\mathcal{G}$ is generated using Grapherator $\mathrm{R}$ package [12] as a random set of 50 planar nodes (samples points from a bi-variate uniform distribution) connected by 137 edges of a Delaunay triangulation. For each of the tested values of $c_{2}$ we started with a graph in a resting state and stimulated the north-most node of the graph. Exemplar time lapses of excitation on $\mathcal{G}$ are shown in Fig. 4 . From the plot of ratio $\zeta$ of edges traversed by excitation wave-fronts versus $c_{2}$ we see that $\zeta$ decreases with increase of $c_{2}$. At $c_{2}=0.111$ we witness a sharp decrease in graph coverage (Fig. 4(d)]. Note that at the same value of $c_{2}$ excitation wave-fronts are no longer entering branches with angle over $90^{\circ}$ (Fig. $2(\mathrm{e})$ and chances of arrest on entering an expansion start to increase exponentially with growth of $c_{2}$ (Fig. $\left.3(\mathrm{~d})\right)$. The coverage $\zeta\left(c_{2}\right)$ can be approximated by two cubic functions: $4125.3-115892 \cdot c_{2}+1.0855 \cdot 10^{6} \cdot c_{2}^{2}-3.3891 \cdot 10^{6} \cdot c_{2}^{3}$ for $c_{2} \in[0.105,0.111]$ and $\left.2442.2-62991\right) \cdot c_{2}+541558 \cdot c_{2}^{2}-1.5519 \cdot 10^{6} \cdot c_{2}^{3}$ for $\left.\left.c_{2} \in\right] 0.111,0.118\right]$. As evidenced by videos of experiments [1] and exemplar plots of activity in Fig. 4(e) the traversing of the $\mathcal{G}$ by excitation wave-fronts is non-linear. For $c_{2}$ below 0.09 we see multiplications of wave-fronts, with some fronts travelling along cyclic routes on the graph. Few outburst of activity, due to multiplication of the fronts for $c_{2}=0.095$ is shown in Fig. $4(\mathrm{e})$. For $c_{2}=0.11$ we witness prolonged periods of low activity with few outbursts of higher aperiodic activity. This is due to a small number of wave-fronts traversing the graph. Some collisions between the wave-fronts lead to annihilation of the fronts, others to formation of additional wave-fronts.

\section{Discussion}

We found how excitability affects a speed of excitation wave-fronts, an ability of the fronts to expand into angled branches, and chances of the fronts to be arrested on entering an expansion. While studying exploration of a random planar graph with excitations we found that the coverage as a function of excitability is a sigmoid function, and that for certain range of excitability values we can observe repeated outburst of excitations due to the wave-fronts travelling along cyclic paths and producing multiple wave-fragments while interacting with each other. The function is comparable with coverage functions obtained in our numerical experiments with two-variable Oregonator model of Belousov-Zhabotinsky (BZ) reaction, where excitability is controlled by parameter $\phi$, and city streets networks are explored by oxidation wave fronts: Barcelona [5], London [6] and Tehran [4] (Fig. 5). Despite different equations used (Oregonator for street networks and FHN for a random planar graph) similarities are striking. In both cases we have a sigmoid function. The function is rather perfect in case of a random graph (Fig. 4(d) and the function is less pronounced, or even distorted, in the case of street networks (Fig. 5). In the case of a random graph the sharp transition starts roughly at the middle, $c_{2}=0.1115$ of the interval $c_{2}=0.105$, full coverage, and $c_{2}=0.118$, no coverage. 


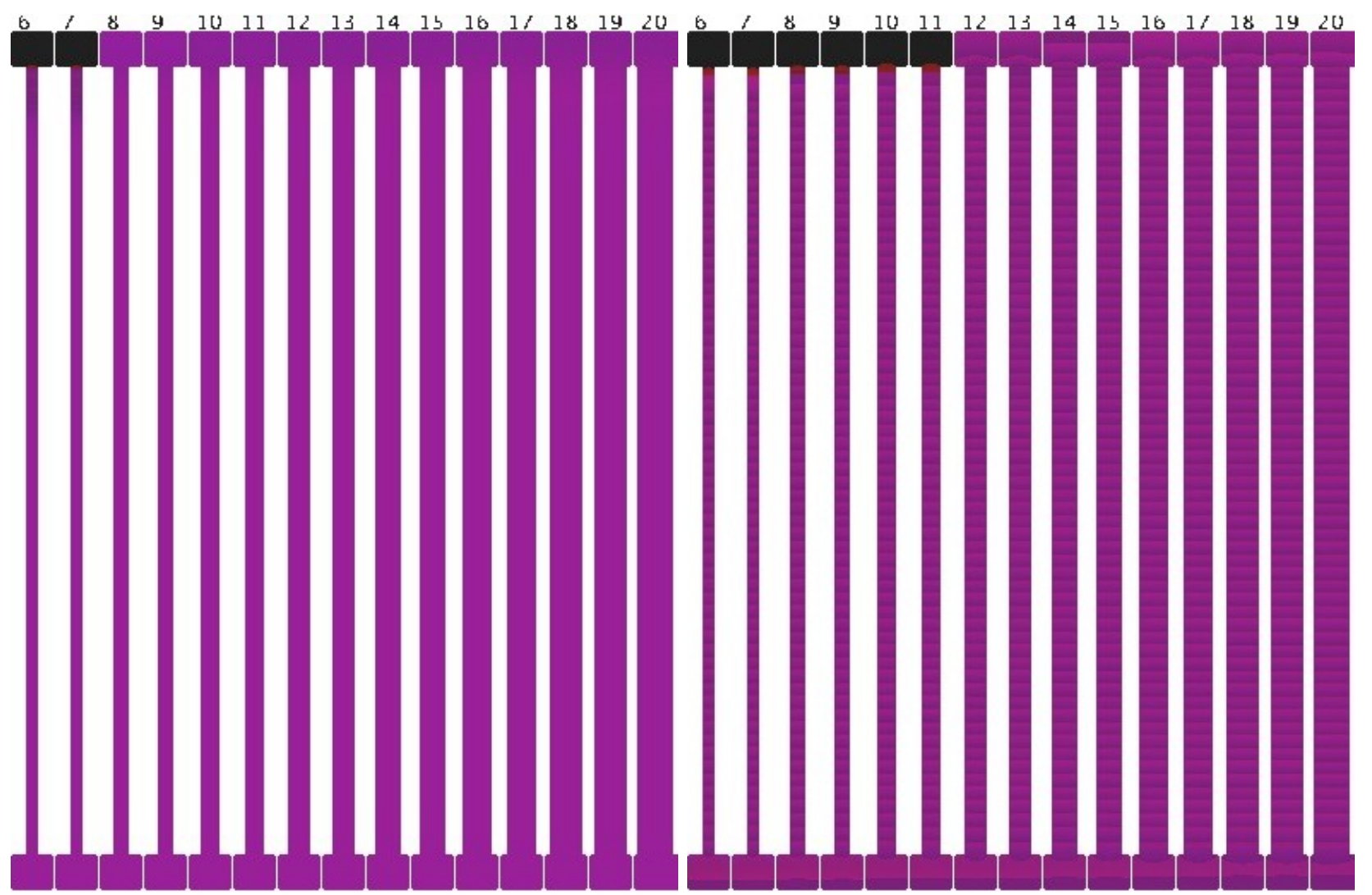

(a) $c_{2}=0.09$

(b) $c_{2}=0.11$

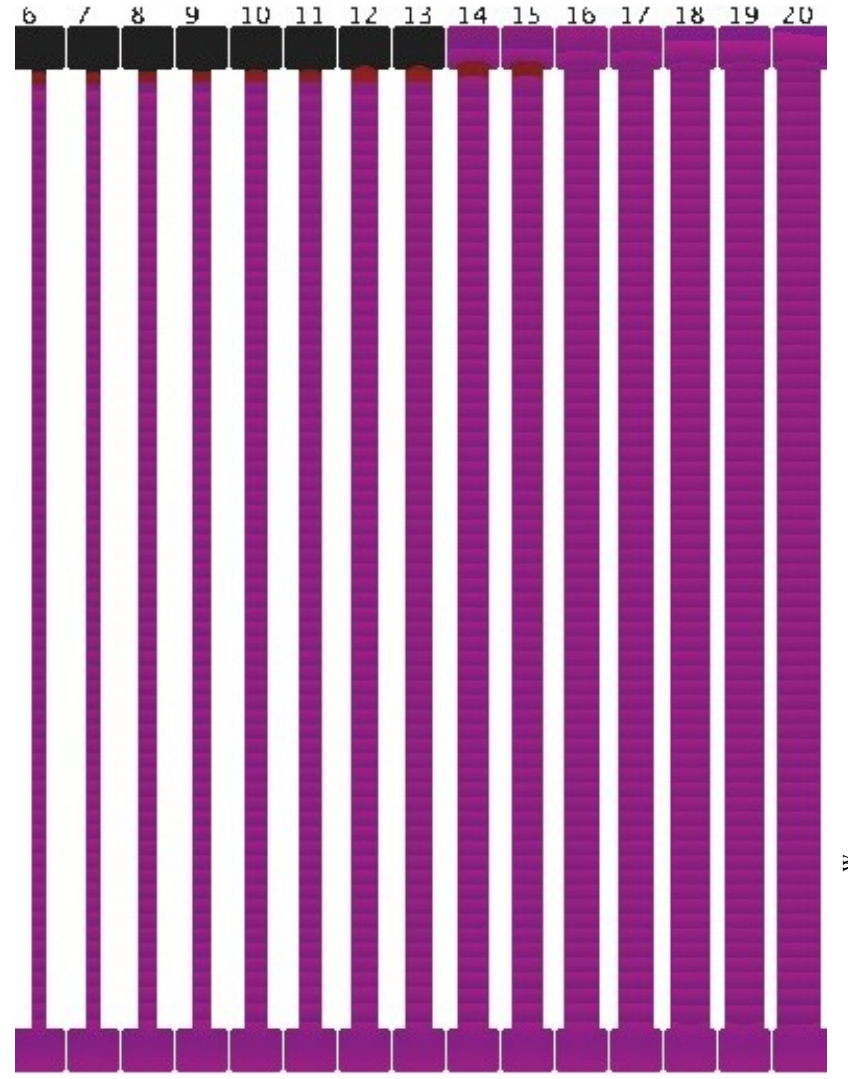

(c) $c_{2}=0.12$

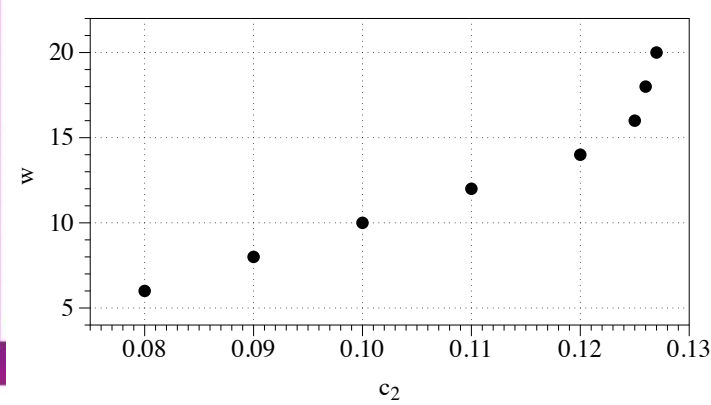

(d)

Figure 3: Arrest on entering expansion. (abc) Exemplar time lapse snapshots, scale 0.4 of the real size. Size of expansion in nodes is shown above each template. (d) $w$ versus $c_{2}$, where $w$ is the smallest width of a channel from which the excitation propagates into the extension reservoir. 


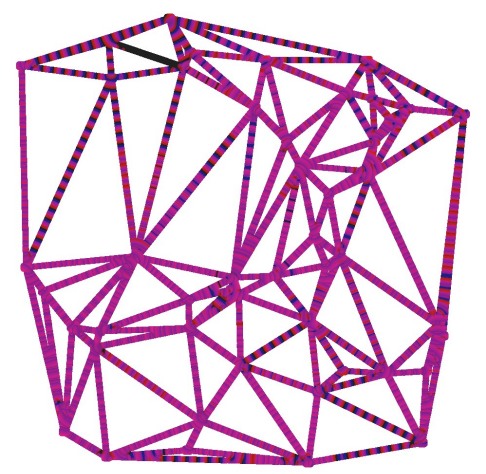

(a) $c_{2}=0.105$

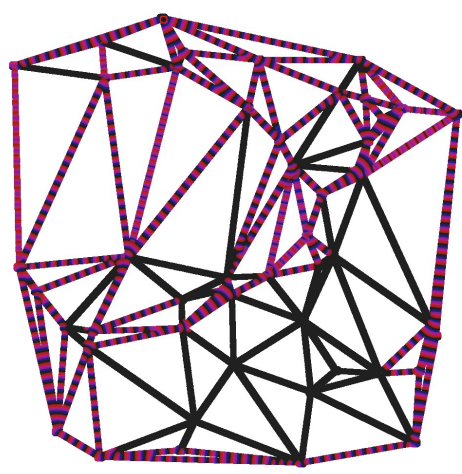

(b) $c_{2}=0.111$

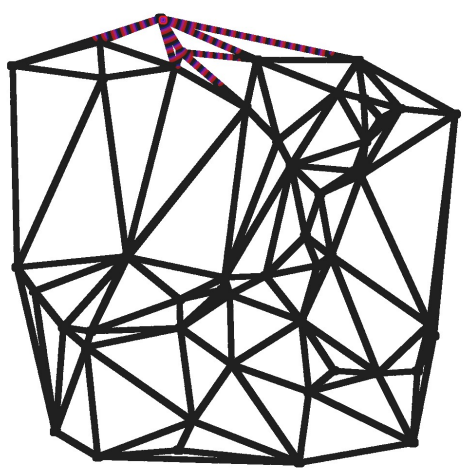

(c) $c_{2}=0.118$

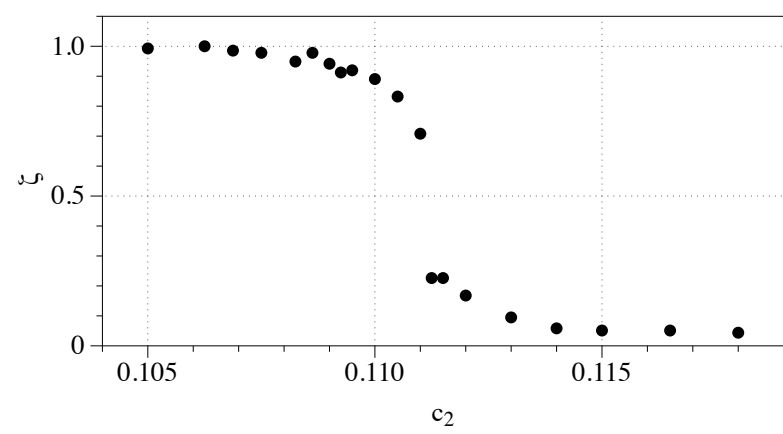

(d)

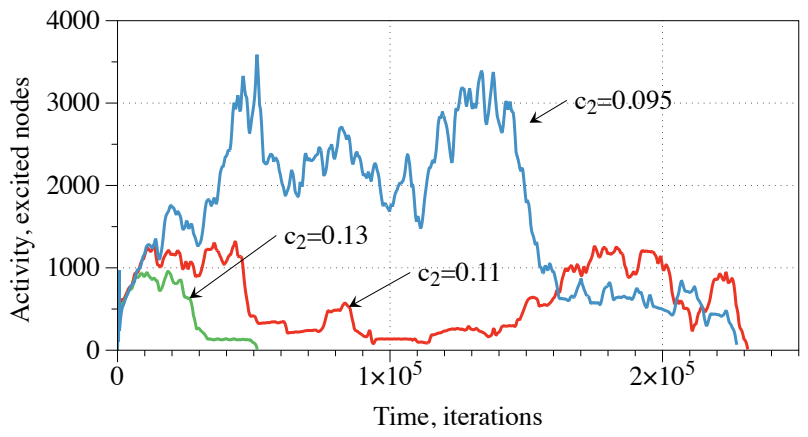

(e)

Figure 4: Exploration of a random planar graph $\mathcal{G}$ by excitation wave-fronts. (a)-(c) Time lapse snapshots of the excitations on $\mathcal{G}$. See videos in [1]. (d) A ratio $\zeta$ of a number of edges traversed by excitation wave-fronts to a total number of edges in $\mathcal{G}$ versus $c_{2}$.

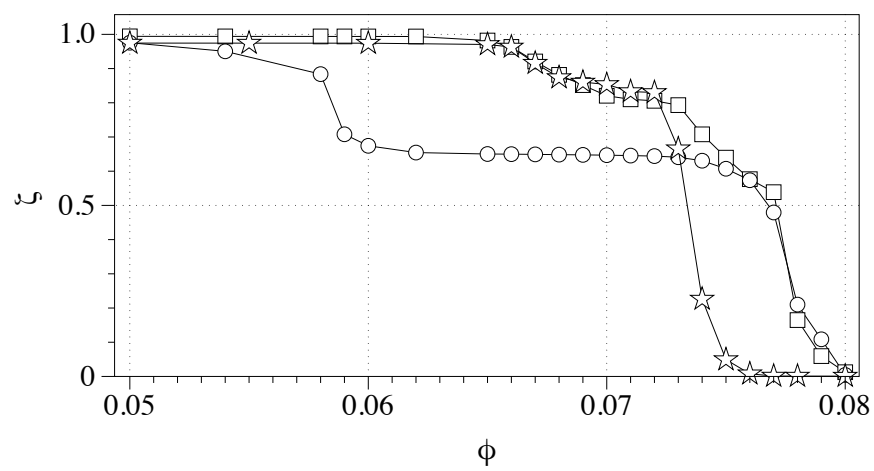

Figure 5: Coverage $\zeta$ of selected fragments of street networks in Tehran (circle), Barcelona (star), London (square) versus excitability $\phi$ of the two-variable Oregonator model of Belousov-Zhabotinsky reaction. See detials in [5]. 
Also, noticeably, the coverage function for Tehran shows two sharp drops in coverage, first just near $\phi=0.06$ and second near $\phi=0.075$. Further studies are required to establish deep relationships between Oregonator BZ and FHN models' behaviour on graphs and their potential for analysing the geometric networks.

\section{References}

[1] Andrew Adamatzky. Supplementary materials. On interplay between geometry and excitability. http://doi.org/10.5281/zenodo.2558149, 2019.

[2] Andrew Adamatzky, Benjamin De Lacy Costello, and Tetsuya Asai. Reaction-diffusion computers. Elsevier, 2005.

[3] Andrew Adamatzky and Benjamin De Lacy Costello. Reaction-diffusion computing. Unconventional Computing: A Volume in the Encyclopedia of Complexity and Systems Science, Second Edition, pages 171-194, 2018.

[4] Andrew Adamatzky and Mohammad Mahdi Dehshibi. Exploring tehran with excitable medium. arXiv preprint arXiv:1807.09023, 2018.

[5] Andrew Adamatzky, Valverdu Jordi, and Georgios Sirakoulis. Chemical excitable medium in barcelona street network as a method for panicked crowds behaviour analysis. Complex Systems, 2019 , in press.

[6] Andrew Adamatzky, Neil Phillips, Roshan Weerasekera, Michail-Antisthenis Tsompanas, and Georgios Ch Sirakoulis. Street map analysis with excitable chemical medium. Physical Review E, 98(1):012306, 2018.

[7] David J Aidley and DJ Ashley. The physiology of excitable cells, volume 4. Cambridge University Press Cambridge, 1998.

[8] Go W Beeler and H Reuter. Reconstruction of the action potential of ventricular myocardial fibres. The Journal of physiology, 268(1):177-210, 1977.

[9] Boris P Belousov. A periodic reaction and its mechanism. Compilation of Abstracts on Radiation Medicine, 147(145):1, 1959.

[10] Omer Berenfeld and Arkady M Pertsov. Dynamics of intramural scroll waves in three-dimensional continuous myocardium with rotational anisotropy. Journal of theoretical biology, 199(4):383-394, 1999.

[11] MS Bingley. Membrane potentials in amoeba proteus. Journal of Experimental Biology, 45(2):251$267,1966$.

[12] Jakob Bossek. A modular multi-step graph generator. https://github.com/jakobbossek/grapherator, 2019.

[13] Gil Bub and Alvin Shrier. Propagation through heterogeneous substrates in simple excitable media models. Chaos: An Interdisciplinary Journal of Nonlinear Science, 12(3):747-753, 2002.

[14] Jorge M Davidenko, Arcady V Pertsov, Remy Salomonsz, William Baxter, and José Jalife. Stationary and drifting spiral waves of excitation in isolated cardiac muscle. Nature, 355(6358):349, 1992.

[15] Roger Eckert and Paul Brehm. Ionic mechanisms of excitation in paramecium. Annual review of biophysics and bioengineering, 8(1):353-383, 1979.

[16] I Farkas, Dirk Helbing, and T Vicsek. Human waves in stadiums. Physica A: statistical mechanics and its applications, 330(1-2):18-24, 2003. 
[17] Illés Farkas, Dirk Helbing, and Tamás Vicsek. Social behaviour: Mexican waves in an excitable medium. Nature, 419(6903):131, 2002.

[18] Richard FitzHugh. Impulses and physiological states in theoretical models of nerve membrane. Biophysical journal, 1(6):445-466, 1961.

[19] Jörg Fromm and Silke Lautner. Electrical signals and their physiological significance in plants. Plant, cell $\&$ environment, 30(3):249-257, 2007.

[20] Xiang Gao, Alexei Krekhov, Vladimir Zykov, and Eberhard Bodenschatz. Initiation of rotors by fast propagation regions in excitable media: A theoretical study. Frontiers in Physics, 6:8, 2018.

[21] Steven S Goldstein and Wilfrid Rall. Changes of action potential shape and velocity for changing core conductor geometry. Biophysical journal, 14(10):731-757, 1974.

[22] LM Gorbunov and VI Kirsanov. Excitation of plasma waves by an electromagnetic wave packet. Sov. Phys. JETP, 66(290-294):40, 1987.

[23] Peter Hammer. Spiral waves in monodomain reaction-diffusion model, 2009.

[24] Helen G Hansma. Sodium uptake and membrane excitation in paramecium. The Journal of cell biology, 81(2):374-381, 1979.

[25] Alan L Hodgkin and Andrew F Huxley. A quantitative description of membrane current and its application to conduction and excitation in nerve. The Journal of physiology, 117(4):500-544, 1952 .

[26] Ch Kittel. Excitation of spin waves in a ferromagnet by a uniform rf field. Physical Review, 110(6):1295, 1958.

[27] Boris Ja Kogan. Excitation wave propagation in narrow passes. In Introduction to Computational Cardiology, pages 199-221. Springer, 2010.

[28] Jan P Kucera, André G Kléber, and Stephan Rohr. Slow conduction in cardiac tissue, ii: effects of branching tissue geometry. Circulation research, 83(8):795-805, 1998.

[29] Pawel Kuklik, Lukasz Szumowski, Prashanthan Sanders, and JJ Żebrowski. Spiral wave breakup in excitable media with an inhomogeneity of conduction anisotropy. Computers in biology and medicine, 40(9):775-780, 2010.

[30] Paweł Kuklik and Jan Jacek Żebrowski. Reentry wave formation in excitable media with stochastically generated inhomogeneities. Chaos: An Interdisciplinary Journal of Nonlinear Science, 15(3):033301, 2005.

[31] Elisa Masi, Marzena Ciszak, Luisa Santopolo, Arcangela Frascella, Luciana Giovannetti, Emmanuela Marchi, Carlo Viti, and Stefano Mancuso. Electrical spiking in bacterial biofilms. Journal of The Royal Society Interface, 12(102):20141036, 2015.

[32] Ann M. McGillviray and Neil A.R. Gow. The transhyphal electrical current of Neuruspua crassa is carried principally by protons. Microbiology, 133(10):2875-2881, 1987.

[33] Jinichi Nagumo, Suguru Arimoto, and Shuji Yoshizawa. An active pulse transmission line simulating nerve axon. Proceedings of the IRE, 50(10):2061-2070, 1962.

[34] Phillip G Nelson and Melvyn Lieberman. Excitable cells in tissue culture. Springer Science \& Business Media, 2012.

[35] Arkady M Pertsov, Jorge M Davidenko, Remy Salomonsz, William T Baxter, and Jose Jalife. Spiral waves of excitation underlie reentrant activity in isolated cardiac muscle. Circulation research, 72(3):631-650, 1993. 
[36] Jack M Rogers and Andrew D McCulloch. A collocation-Galerkin finite element model of cardiac action potential propagation. IEEE Transactions on Biomedical Engineering, 41(8):743-757, 1994.

[37] Sitabhra Sinha, Kenneth M Stein, and David J Christini. Critical role of inhomogeneities in pacing termination of cardiac reentry. Chaos: An Interdisciplinary Journal of Nonlinear Science, 12(3):893-902, 2002.

[38] JC Slonczewski. Excitation of spin waves by an electric current. Journal of Magnetism and Magnetic Materials, 195(2):L261-L268, 1999.

[39] Kazimierz Trebacz, Halina Dziubinska, and Elzbieta Krol. Electrical signals in long-distance communication in plants. In Communication in plants, pages 277-290. Springer, 2006.

[40] M Tsoi, AGM Jansen, J Bass, W-C Chiang, M Seck, V Tsoi, and P Wyder. Excitation of a magnetic multilayer by an electric current. Physical Review Letters, 80(19):4281, 1998.

[41] Wei Wang, Shanzhuo Zhang, Haibo Ni, Clifford J Garratt, Mark R Boyett, Jules C Hancox, and Henggui Zhang. Mechanistic insight into spontaneous transition from cellular alternans to arrhythmia - a simulation study. PLoS computational biology, 14(11):e1006594, 2018.

[42] LD Weise and AV Panfilov. Emergence of spiral wave activity in a mechanically heterogeneous reaction-diffusion-mechanics system. Physical review letters, 108(22):228104, 2012.

[43] AM Zhabotinsky. Periodic processes of malonic acid oxidation in a liquid phase. Biofizika, 9(306-311):11, 1964.

[44] Anatol M Zhabotinsky. Belousov-zhabotinsky reaction. Scholarpedia, 2(9):1435, 2007.

[45] Matthias R Zimmermann and Axel Mithöfer. Electrical long-distance signaling in plants. In Long-Distance Systemic Signaling and Communication in Plants, pages 291-308. Springer, 2013.

[46] Vladimir S Zykov and Eberhard Bodenschatz. Wave propagation in inhomogeneous excitable media. Annual Review of Condensed Matter Physics, 9:435-461, 2018. 